\title{
Die Problematik des Begriffes hebraica veritas
}

\author{
James Alfred Loader \\ Institut für Alttestamentliche Wissenschaft \\ und Biblische Archäologie, Universität Wien \\ und Professor Extraordinarius UNISA, Pretoria
}

\begin{abstract}
The formal problematic of the concept hebraica veritas

Proceeding from the importance of the concept of hebraica veritas in terms of both its original intention and of the opposing positions on Holy Scripture entertained by the Roman Catholic tradition and the emerging Protestant views during the Reformation, a brief discussion of the meaning and early context of the concept is given. The formal problematic of the hebraica veritas as found in the Tanak is addressed vis-à-vis its latinised version in the Greek text tradition. Jerome's use of the concept is analysed on the basis of his textual justification for it. Pneumatological and salvation-historical dimensions are identified, and the function of the concept as self-identification over against Judaism is discussed, as well as its implications for delimiting the canon. It is concluded that the concept needs to be foregrounded anew in light of its significant impact in the context of accounting for the concepts of Holy Scripture, canon and therefore canon-based endeavours to construe a "biblical theology" of the "whole Bible".
\end{abstract}

\section{EINLEITUNG}

Nach dem vom Kirchenvater Sophronius Eusebius Hieronymus (347419 n.Chr.) stammenden Begriff hebraica veritas, "die hebräische Wahrheit", liege die Wahrheit nur im hebräischen Text der rabbinischen Bibelüberlieferung (Tanak) vor, weil - gegenüber der im 4. Jh. n.Chr. recht mannigfaltigen griechischen Überlieferung der Septuaginta - nur er der alttestamentliche Text der Verfasser des Neuen Testaments 
gewesen sei. "Wahrheit" bezieht sich auf die hebräisch von Gott an Mose und die Propheten offenbarte Wahrheit, die damit die ausschließliche Norm für die Wahrheit der kirchlichen Lehre sei, während die griechische Überlieferung zwar gelesen, nicht aber für die Begründung der christlichen Lehre verwendet werden dürfte. Daher ist der Begriff der "hebräischen Wahrheit" durchaus ein christlicher, und darüber hinaus, wie unten zu sehen ist, in bewusstem Gegensatz zur jüdischen Tradition.

\section{DIE FUNDIERUNG DER HEBRAICA VERITAS BEI HIERONYMUS}

Hieronymus prägte den Begriff spezifisch von seinem 391 bis 405 n.Chr. durchgeführten Projekt zur Übersetzung des Alten Testaments ins Lateinische. Nach inm waren sowohl der Text des Tanak als auch sein Umfang Ausdruck der wahren Offenbarung Gottes an Israel, daher konnte nur diese Form des Alten Testaments die Wahrheit erhalten. Folgerichtig betrachtete er sie als Bibel der Verfasser des Neuen Testaments und Norm für Lehre und Leben. Untersucht man die Vorreden zu den verschiedenen Büchern in der Vulgata, kommt eine apologetische, manchmal auch recht polemische Verteidigung seiner Auffassung der "hebräischen Wahrheit" vor. An verschiedenen Stellen ${ }^{1}$ bietet er Argumentationen für diese Sicht, in denen eine empirische, eine heilshistorische und eine pneumatologische erkennbar sind. Ich beginne mit den empirischen Überlegungen von Hieronymus, und zwar mit Überlegungen aus dem Prologus in Pentateucho ${ }^{2}$ von ca. 400 n.Chr.

\subsection{Die empirische Begründung der hebraica veritas}

Der empirische Aspekt in der Argumentführung des Hieronymus wird gleich am Anfang seiner Vorrede deutlich:

[8] Quod ut auderem, Origenis me studium provocavit, qui editioni antiquae translationem Theodotionis miscuit, asterisco et obelo, id est stella et veru, opus omne distinguens, dum aut inlucescere facit quae minus ante fuerant aut superflua quaeque iugulat et confodit,

\footnotetext{
${ }^{1} \mathrm{Vgl}$. die unten angeführten und besprochenen Texte, die repräsentativ gewählt wurden.

${ }^{2}$ Zitiert nach Biblia Sacra iuxta Vulgatam Versionem in der Württembergischen Ausgabe von Robert Weber et al, 1969.
} 
maximeque Evangelistarum et Apostolorum auctoritas, in quibus multa de Veteri Testamento legimus quae in nostris codicibus non habentur, ut est illud: "Ex [13] Aegypto vocavi filium meum", et: "Quoniam Nazareus vocabitur", et: "Videbunt in quem conpunxerunt", et: "Flumina de ventre eius fluent aquae vivae", et: "Quae nec [15] oculus vidit, nec auris audivit, nec in cor hominis ascenderunt quae praeparavit Deus diligentibus se", et multa alia quae

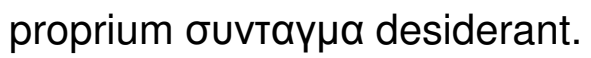

[8] Dass ich es wagte [sc., zu übersetzen], dazu hat mich das Studium von Origenes provoziert, der der Übersetzung des Theodotion die alten Ausgaben mit Asterisk und Obelus, also mit Stern und Spieß beigemischt hatte, ein Werk, das alles hervorhebt, da er entweder das vormals Unauffallende aufleuchten lässt oder das Überflüssige ersticht und durchbohrt, besonders aber die Autorität der Evangelisten und Apostel, bei denen wir vieles vom Alten Testament lesen, das wir in unseren Kodexen nicht haben, wie folgendes: "Aus [13] Ägypten habe ich meinen Sohn gerufen", und: "Der Nazoräer genannt wird", und: "Sie werden sehen, in wen sie gestochen haben", und: "Ströme von lebendigem Wasser werden von seinem Inneren sprudeln", und: "Was weder ein [15] Auge gesehen, noch ein Ohr gehört hat oder im Herzen eines Menschen aufgekommen ist, hat Gott vorbereitet für diejenigen, die inn lieben", und vieles andere, das einen geeigneten Kontext verlangt.

Dieses Argument soll darlegen, dass die Autoren des Neuen Testaments das Alte Testament nach dem hebräischen Text zitieren. Der Duktus ist, dass die Apostel ${ }^{3}$ der Septuaginta nicht genau folgen, und dass der hebräische Text demgemäß die Bibel der neutestamentlichen Autoren sein muss.

Angeführt werden folgende Texte, die Hieronymus etwas weiter im Prolog (Zeilen 18-19) jeweils einem Buch des Alten Testaments zuweist:

- $\quad$ Matthäus 2,15, wo aus Hosea 11,1 zitiert wird: "Aus Ägypten habe ich meinen Sohn gerufen". Im griechischen Text von

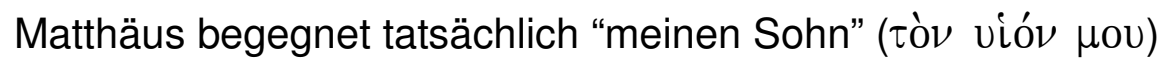
im Akkusativ Singular gemäß dem hebräischen Text, wo das Suffix der ersten Person verwendet wird (לבְני), und nicht "seine

\footnotetext{
${ }^{3}$ Vgl. Prologus in Libro Paralipomenon, 22ff.; Prologus in Ezram $21 \mathrm{ff}$. , wo Hieronymus ähnlich argumentiert.
} 


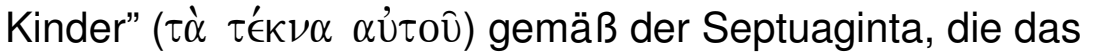
Substantiv im Plural und das pronominale Element in der dritten Person hat. In diesem Fall steht der neutestamentliche Text in der Tat der hebräischen Textform näher als der Septuaginta. Andererseits ist jedoch auch darauf hin zu weisen, dass Matthäus die Wiedergabe der Septuaginta ohnehin nicht verwenden konnte, da die Pluralform der Septuaginta ("Kinder") für die Anwendung auf Jesus als Einzelperson ungeeignet ist.

- $\quad$ Matthäus 2, 23: “... damit erfüllt würde, was gesagt ist durch die Propheten: Er soll Nazoräer heißen”. Angesichts des Verweises auf "die Propheten" im Plural, muss damit die Erfüllung der allgemeinen prophetischen Botschaft gemeint sein, wobei Jesaja 11, 1 der Aufhänger ist. Matthäus verbindet zu diesem Zweck volksetymologisch die Wurzel נצר und נזר, um das erste als "Nazoräer", "aus Nazaret kommend" und das zweite als "Naziräer", "geweihter" mit einander zu identifizieren. Da Hieronymus in Zeile 19 seines Prologs behauptet, dieses Zitat stünde im Buch Jesaja, muss er wie Matthäus auf Jesaja 11, 1 zielen, wo das hebräische Wort für "Reis" oder "Zweig" im

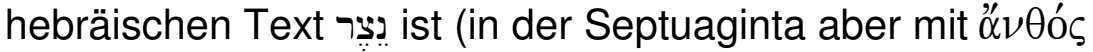

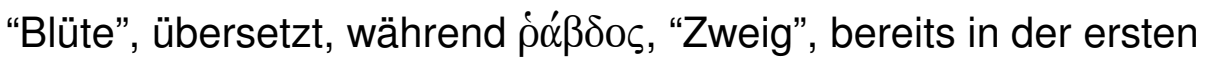
Vershälfte vorkommt) und damit von der Wurzel her mit dem Wort für "Naziräer" (נִִיר) vergleichbar ist. Abgesehen davon, dass die Wurzeln unterschiedlich sind ( $¥$ und i) und somit von der Sprachform her kein Argument im Sinne des Hieronymus ermöglichen, ist auch sonst keine Stelle in Jesaja oder anderswo im Alten Testament zu finden, die das Zitat enthält - weder in der Septuaginta, noch im hebräischen Alten Testament. Auch wenn Jesaja 11,1 hier angedacht ist, kann das Zitat noch immer nicht als Beleg für die Nähe des Neuen Testaments zum hebräischen Text angeboten werden. Wenn allerdings überlegt wird, dass es als allgemeine Paraphrase der generellen prophetischen Botschaft gemeint ist (wie in Esra 9, 11, wo ebenfalls Worte mehrerer Propheten angeboten werden als seien diese ein Zitat, das es aber im Alten Testament nicht gibt), dann belegt es gerade nicht die Zuverlässigkeit des hebräischen Textes gegenüber der Septuaginta. Vielmehr wird klar, dass es bei Matthäus umgekehrt um eine sehr freie Verwendung von ähnlich klingenden Vokabeln im Alten Testament geht. 
- Johannes 19, 37: "Und wiederum sagt die Schrift an einer anderen Stelle: 'Sie werden den sehen, den sie durchbohrt

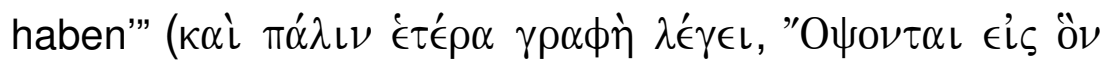
${ }^{\epsilon} \xi \in \kappa \in \in(\in \tau \eta \eta \sigma \alpha \nu)$. Das Zitat ist aus Sacharja 12, 10: "Und sie werden mich ansehen, den sie durchbohrt haben". Es bezieht sich eindeutig auf eine spezifische Bibelstelle, und auch hier steht die neutestamentliche Form dem hebräischen Text keineswegs näher als der Septuaginta. Im hebräischen Text steht das Suffix 1. Person Singular bei der Präposition: "und sie werden mich,

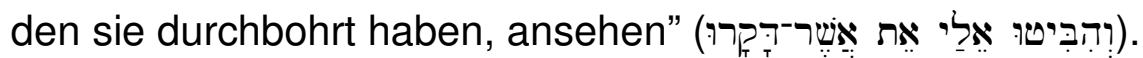

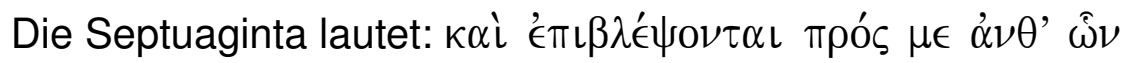

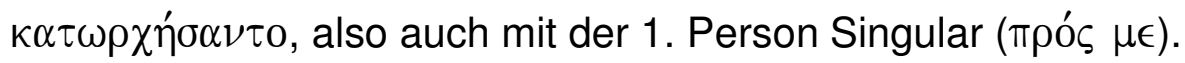
Anders als von Markschies behauptet, ${ }^{4}$ stimmen der Tanak und die Septuaginta gegenüber dem Neuen Testament überein, so dass vom neutestamentlichen Gebrauch her keine Folgerung auf die Überlegenheit des hebräischen Textes im Sinne von Hieronymus möglich ist.

- Johannes 7, 38 heißt: “ ... wie die Schrift sagt, von dessen Leib werden Ströme lebendigen Wassers fließen" ( $\kappa \alpha \theta \dot{\omega} \varsigma \in \hat{\imath} \pi \epsilon \nu \dot{\eta}$

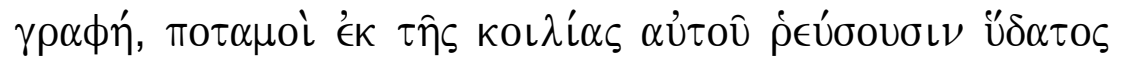
$\zeta \hat{\omega} \nu \tau 0 \varsigma)$. Wiederum findet sich das Zitat weder im hebräischen Text des Alten Testaments noch in der Septuaginta. Da Hieronymus das Zitat aber in Zeile 18/19 seines Prologs dem Proverbienbuch zuschreibt, hält er am wahrscheinlichsten Sprüche 18,4 für den Bezugsort des Evangeliums: "Tiefes Wasser sind die Worte vom Munde eines Mannes, ein

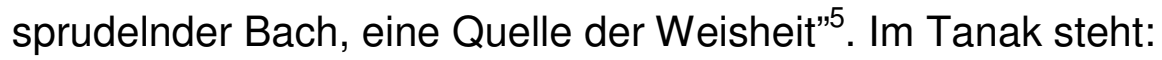

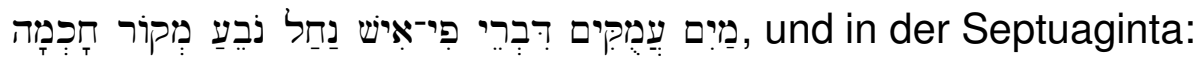

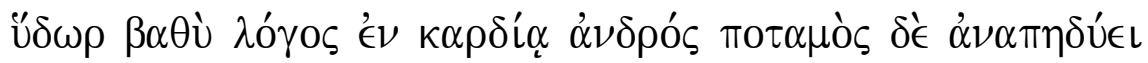
$\kappa \alpha \grave{\imath} \pi \eta \gamma \grave{\eta} \zeta \omega \eta \bar{\varsigma}$. Hier ist der neutestamentliche Vers der Septuaginta sogar näher als dem hebräischen Text, indem in den beiden griechischen Logien von "lebendigem Wasser" bzw. "Wasserströmen des Lebens" die Rede ist, während der hebräische Wahrspruch von einer "Quelle der Weisheit" redet.

\footnotetext{
${ }^{4}$ Markschies 1994:157, Anm. 148.

${ }^{5}$ Vgl. Spr. 5,16; Ez. 36,25; Joel 3,1; HId. 4,15; Str.-B. 2,492.
} 
- $\quad$ 1. Korinther 2, 9: "Sondern, wie geschrieben steht: ,Was kein Auge gesehen hat und kein Ohr gehört hat und in keines Menschen Herz gekommen ist, was Gott bereitet hat denen, die

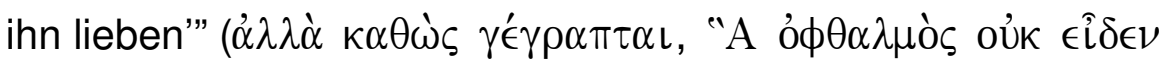

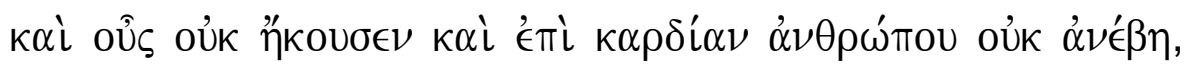

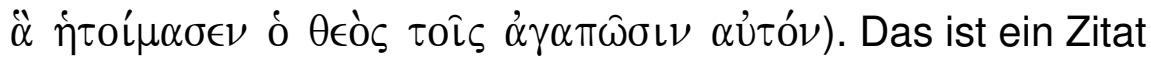
aus Jesaja 64,3[4], wo der Tanak folgendermaßen lautet: "Und was von alters her nicht vernommen ist, nicht gehört war, was kein Auge gesehen hat - einen Gott außer dir, der so wohl tut dem, der auf inn harrt".

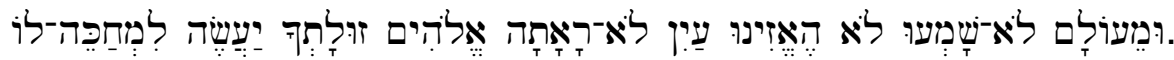
Die Septuaginta: "Von alters her haben wir nicht gehört und unsere Augen haben nicht gesehen - einen Gott außer dir, und deine Werke, die du tun wirst denen, die auf Gnade harren" (⿳亠丷厂т⿱丶万⿰丨丶

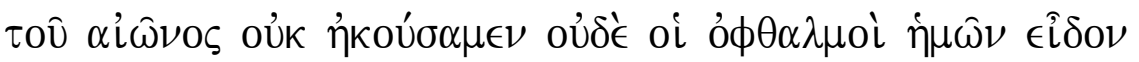

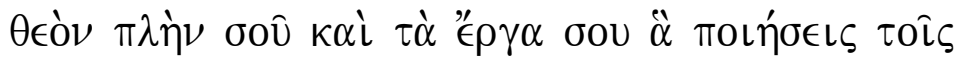

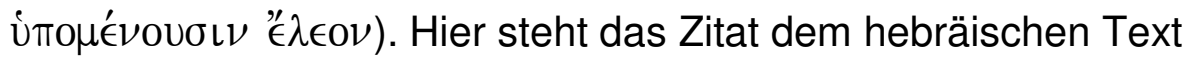
in einer Hinsicht etwas näher, weil beide gegenüber der Septuaginta "Auge" im Singular haben, aber andererseits verwenden der hebräische Text und die Septuaginta das Verb "harren", wo Paulus "lieben" verwendet, während die Septuaginta, nicht aber Paulus, מעוֹלוֹם wiedergibt.

Das Argument, das Hieronymus auf spezifische Belege versucht aufzubauen, ist also auf keinen Fall schlüssig, da die von ihm als Beweismaterialien verwendeten Zitate im Neuen Testament dem hebräischen Text nur ungenau folgen, ${ }^{6}$ jedenfalls nicht genauer als die Wiedergabe dieser Stellen in der Septuaginta. Das legt nahe, dass sich die hebraica veritas vielmehr auf a priori Voraussetzungen stützt, die Hieronymus versucht, nachträglich mit textfundierten Ausführungen annehmlich zu machen.

Dass die hebraica veritas für Hieronymus mehr notwendige Voraussetzung als Praxis war, wird aus einem anderen Blickwinkel

\footnotetext{
${ }^{6}$ So auch Bammel (1988:145), der auf weitere Behauptungen des Hieronymus verweist (etwa in De optimo genere interpretandi, Ep. 57,7ff.). Danach leugnet Hieronymus nicht, dass das Neue Testament die Septuaginta verwende, macht aber geltend, dass das nur dort geschehe, wo diese vom hebräischen nicht abweicht. Auch die Beispiele, die Markschies in seiner Diskussion von Ep. 57 behandelt, retten die These von Hieronymus nicht, da nicht einmal alle, die Markschies im Sinne von Hieronymus akzeptiert, so stimmen (s. etwa oben zu Joh. 19,37). Daher muss Markschies (1994:157, vgl 160) auch zugeben, dass dies "eine bestürzende Folge für seine Konsistenz" hat.
} 
bestätigt, und zwar durch einen Vergleich seines Vorgehens beim Übersetzen und seiner grundsätzlichen Aussage zum Grundprinzip dieser Arbeit. Es heißt in seinem "Buch zur besten Art des Übersetzens" 7 :

Ego enim non solum fateor, sed libera voce profiteor me in interpretatione Graecorum absque scripturis sanctis, ubi et verborum ordo mysterium est, non verbum e verbo, sed sensum exprimere de sensu.

Aber ich gestehe nicht nur, sondern bekenne mich freilich und laut zur Übersetzung der Griechen, aber mit Ausnahme der heiligen Schriften, wo sogar die Ordnung der Worte ein Mysterium ist, nicht Wort für Wort, sondern Sinn aus Sinn wiederzugeben.

Der Kontext ist die Verteidigung seines Vorgehens beim Übersetzen, also die Verantwortung für seine grundsätzlichen Prinzipien. Es heißt, dass sinngemäße Übersetzung (sensum exprimere de sensu) besser als wörtliche Übersetzung (verbum e verbo) sei. Wenn das der Grundsatz ist, ist die Ausnahme umso wichtiger: "die Heiligen Schriften, wo sogar die Ordnung der Worte ein Mysterium ist". 8 Die Sonderstellung der Bibel erscheint in einer pauschalen Aussage, die zur Überzeugung passen würde, dass die Wahrheit der göttlichen Mysterien bis in die feinsten Details von Syntax und Wortfolge des hebräischen Textes ausgedruckt wird, also zu einer wirklich konsequenten Auffassung der hebraica veritas. Mir scheint das gegen Markschies' starke Betonung einer philologischen gegenüber einer theologischen Begründung der hebraica veritas ${ }^{9} \mathrm{zu}$ sprechen. Hier wird gerade in einem Absatz zur allgemeinen philologischen Arbeit eine Ausnahme für das hebräische Alte Testament behauptet, und zwar aufgrund der in der hebraica veritas ausgedrückten Mysterien Gottes, was wiederum nur aufgrund einer Inspirationsauffassung möglich ist.

\footnotetext{
${ }^{7}$ Ad Pammachium de optimo genere interpretandi. Text: Bartelink (1980:13). Hervorhebung von mir.

${ }^{8}$ Sparks (1970:523), betont auch die Bedeutung der Ausnahme.

${ }^{9}$ Markschies (1994:131-181, passim; vgl. besonders S. 146), wo es heißt, die Wende zur hebraica veritas sei "durch eine gründliche philologische Arbeit zum Alten Testament und nicht durch eine textferne theologische Entscheidung zur Inspirationstheorie ausgelöst". Auch wenn Hieronymus' philologische Ausbildung inn dafür vorbereitete (was ich nicht bestreiten möchte), ändert sich dadurch die theologische Dimension der hebraica veritas nicht. Jedenfalls ist eine "textfernere" Aussage dazu kaum denkbar.
} 
Das Prinzip deckt sich aber nicht mit der aktuellen Praxis der Bibelübersetzung des Hieronymus. ${ }^{10}$ Manchmal übersetzt er sklavisch buchstäblich, manchmal aber unter dem Einfluss der Vetus Latina, der Septuaginta, jüdischer Folklore (z.B. 1. Sam. 17,4.23), oder christlicher Lesarten (z.B. Hab. 3,18). Er vermeidet gerne die Verwendung des gleichen lateinischen Wortes, manchmal in einem spezifischen Abschnitt, aber manchmal auch massiv (z.B. die unterschiedlichen Übersetzungen von דבר in Jeremia und Ezechiel), manchmal übersetzt er Personennamen, manchmal nicht, manchmal schiebt er Glossen ein, usw. ${ }^{11}$

Aus diesen Gründen konnte Sparks die Vulgata zu Recht "eine merkwürdige Mischung" ("curious mixture") nennen. Dieses Vorgehen passt aber kaum zur prinzipiellen Überzeugung, dass der hebräische Text bis in seine sprachlichen Erscheinungsformen die Wahrheit der göttlichen Mysterien ausdrückt. Wieder sehen wir, dass der apriorische Charakter von Hieronymus' Auffassung der hebraica veritas und sein tatsächlicher Umgang mit dem hebräischen Text auseinander klaffen. Für den Vorzug des hebräischen Textes muss es also eine andere Erklärung als Hieronymus' detaillierter Umgang mit dem hebräischen Tanak selbst geben. Diese Erklärung wird uns durch zwei weitere Argumente im Prolog-Text geboten. Zu diesen wenden wir uns demnächst.

\subsection{Der pneumatologische Aspekt der hebraica veritas}

Das zweite Element im Vorwort zum Pentateuch bezieht sich auf den Unterschied zwischen der Inspiration der hebräischen Bibel und der griechischen Übersetzung der Septuaginta:

Denique ubicumque sacratum aliquid [24] Scriptura testatur de Patre et Filio et Spiritu Sancto, aut aliter interpretati sunt aut omnino tacuerunt, ut et regi satisfacerent et arcanum fidei non vulgarent. ... et multo post tempore losepphus nihil tale rettulerint, sed in una basilica congregatos contulisse scribant, non prophetasse. Aliud est enim vatem, aliud esse interpretem: ibi spiritus [30] ventura praedicit, hic eruditio et verborum copia ea quae intellegit transfert

Dementsprechend, überall wo etwas Heiliges in der [24] Schrift über den Vater, den Sohn und den Heiligen Geist bezeugt wird, haben sie es entweder anders übersetzt oder

\footnotetext{
${ }^{10}$ Bartelink (1980:4).

${ }^{11}$ Vgl. Sparks (1970:525-526).
} 
verschwiegen, damit sie den König zufrieden stellen konnten und zugleich das Geheimnis des Glaubens nicht allgemein bekannt geben mussten. ... und viele nach der Zeit von Josephus haben nichts dieser Art berichtet, sondern, dass sie sich in einer Basilika versammelten und schrieben, nicht aber prophezeiten. Denn es ist eines, Seher zu sein, ein anderes, Dolmetscher zu sein: dort sagt der Geist voraus [30] was kommt, hier übersetzt man durch Gelehrsamkeit und Reichtum an Worten, was man verstanden hat ...

Es ist nach Hieronymus undenkbar, dem heiligen Geist zuzumuten, die Arbeit der Übersetzung inspiriert zu haben. Die Tätigkeit der Siebzig war demnach keine Prophetie, sondern gelehrter Umgang mit Worten. Hingegen ist der hebräische Text Niederschlag der vorhersagenden Inspiration des Heiligen Geistes.

So argumentiert Hieronymus im Prolog zum Buch Jesaja:

[6] Ac primum de Isaia sciendum quod in sermone suo disertus sit, quippe ut vir nobilis et urbanae elegantiae nec habens quicquam in eloquio rusticitatis admixtum. Unde accidit, ut prae ceteris florem sermonis eius translatio non potuerit conservare. [9] Deinde etiam hoc adiciendum, quod non tam propheta dicendus sit quam evangelista. Ua enim universa Christi Ecclesiaeque mysteria ad liquidum persecutus est, ut non eum putes de futuro vaticinari, sed de praeteritis historiam texere. Unde conicio [12] noluisse tunc temporis Septuaginta interpretes fidei suae sacramenta perspicue ethnicis prodere, ne sanctum canibus et margaritas porcis darent, quae, cum hanc editionem legeritis, ab illis animadvertetis abscondita.

[6] Und zunächst ist von Jesaja zu wissen, was in seiner Sprache erörtert ist; sicher hat er als edler Mann von kultivierter Eleganz keine Grobheit in die Redekunst gemischt. Daher passiert es, dass im Vergleich mit anderen die Übersetzung die Blüte seiner Sprache nicht beibehalten konnte. [9] Weiterhin ist noch das beizufügen, das es nicht sosehr von einem Propheten als von einem Evangelisten gesprochen wird. Denn alle Mysterien Christi und der Kirche werden bis zu solcher Klarheit verfolgt, dass du nicht denken würdest, sie seien von der Zukunft geweissagt, sondern dass sie von der Vergangenheit eine Geschichte zusammenwebten. Daher behaupte ich, [12] dass die Siebzig Übersetzer damals nicht bereit waren, die Sakramente ihres Glaubens den Heidenvölkern durchsichtig 
vorzulegen oder das Heilige den Hunden und die Perlen den Schweinen zu geben; diese Dinge, wirst du bemerken als du diese Ausgabe gelesen hast, wurden durch sie verborgen.

Obwohl er das Substantiv "Inspiration" oder verwandte Wortformen nicht verwendet, verbindet Hieronymus hier zwei Aspekte einer klaren Inspirationsauffassung: Auf der einen Seite spricht der Prophet nicht so sehr als Prophet, sondern vielmehr als Evangelist, so dass er die Mysterien Christi und der Kirche so klar wiedergibt, als wäre das ein historischer Bericht. Der Prophet wusste also vom Kommenden als wäre es Vergangenes. Das ist nur durch die Offenbarung einer unmittelbaren göttlichen Inspiration möglich. Auf der anderen Seite behält der hebräisch sprechende Prophet seinen normalen Stil, so dass man seine Kultiviertheit und Bildung deutlich in seinem Sprachgebrauch spüren kann. Das heißt, dass die Inspiration Erkenntnis der christlichen Mysterien das normale Bewusstsein des Propheten und seine Fähigkeiten nicht aufhebt. ${ }^{12}$

Das verwendet Hieronymus nun, um seine Behauptung der "abweichenden" Qualität der Septuaginta zu untermauern: die Siebzig wollten die "Heiligkeiten" (sacramenta) ihres Glaubens nicht an Heiden preisgeben und haben "das Heilige" (sanctum) deswegen mit einer undeutlichen Übersetzung "verhüllt" (abscondita). Dieses Argument fügt sich aber schwerlich in die Intention seines Verwenders. Wenn Jesaja die christlichen und kirchlichen "Mysterien" so klar dargestellt hätte, dass sie wie das Evangelium des bereits Geschehenen erschienen, und wenn die Übersetzer der Septuaginta "daher" (unde) die Mysterien als Perlen verbergen wollten, dann wäre zu erwarten, dass sie den klaren Sinn der "christlich-kirchlichen" Verkündigung Jesajas verstanden hätten. Das würde bedeuten, dass sie inspiriert waren - genau das, was Hieronymus in Abrede stellte. Das Argument, wie es tatsächlich geführt wird, unterminiert sich selbst: Die Siebzig waren als Übersetzer nur uninspirierte Interpreten ihres nichtchristlichen jüdischen Glaubens (interpretes fidei suae), haben also nicht den unverkennbar klaren christlichen Inhalt Jesajas verstanden. Andererseits müssen sie aber doch den Inhalt verstanden haben, jedenfalls genug davon, um inn vor den Heiden zu verbergen.

\footnotetext{
${ }^{12}$ Ähnliche Voraussetzungen kommen auch in den Prologen zu Jeremia und Amos vor (vgl. Kelly 1977:63). Dass im Jesaja-Prolog eine deutliche theologische Dimension vorliegt, muss auch Markschies (1994:152) zugeben, obwohl er das nicht weiter verfolgt. Er hätte hier auch darauf hinweisen können, dass Hieronymus auf die stilistische Eigenständigkeit der inspirierten Propheten besteht und damit seiner philologischen Bildung gerecht wird, scheint mir aber die Gelegenheit zu verpassen.
} 
Die logische Verbindung, die durch unde hergestellt wird, untergräbt die Beweiskraft der eigenen Ausführungen. ${ }^{13}$

Damit wird die prinzipielle Überzeugung des Hieronymus hervorgehoben: Die Verfasser der Bibel des Alten sowie des Neuen Testaments wussten unter der göttlichen Inspiration von den christlichen Wahrheiten, die sie bei vollem Bewusstsein ${ }^{14}$ im eigenen Stil formulierten. Dazu waren die jüdischen Übersetzer der Septuaginta aus der Zeit zwischen den beiden Testamenten aber nicht fähig. Seine Inspirationsauffassung ist also der eine Aspekt in der theologischen Fundierung der hebraica veritas.

\subsection{Der heilshistorische Aspekt der hebraica veritas}

Hieronymus führt auch ein weiteres Argument an, diesmal auf heilshistorischer Ebene: ${ }^{15}$

IIli [36] interpretati sunt ante adventum Christi et quod nesciebant dubiis protulere sententiis, nos post passionem et resurrectionem eius non tam prophetiam quam historiam scribimus; aliter enim audita, aliter visa narrantur: quod melius intellegimus, melius et [39] proferimus.

Diese [36] interpretierten vor dem Advent Christi, und was sie nicht wussten, übersetzten sie in zweifelhaften Sätzen, wir schreiben aber nach seinem Leiden und seiner Auferstehung, nicht so sehr Prophetie als vielmehr Geschichte; denn in dem einen wird erzählt, was gehört wurde, in dem anderen, was gesehen wurde: was wir besser verstehen, [39] das übersetzen wir auch besser.

Hier kommen wiederum keine empirischen Verweise auf spezifische Texte vor, daher auch keine induktive Begründung der eigenen These auf deren Grundlage, sondern eine deduktive Argumentation aufgrund eines für allgemein gültig gehaltenen Theologumenon. Die Übersetzer

\footnotetext{
${ }^{13}$ Demgegenüber hat Augustin diesen logischen Fehler nicht gemacht, als er genau das Gegenteil von der Inspiration der Siebzig behauptete (De Civitate Dei XVIII,43): die Übersetzer der Septuaginta waren genauso inspiriert wie die Propheten Israels. Dieser Punkt kommt unten (§ 2.3) im Rahmen der ante-adventum-Argumentführung von Hieronymus wieder vor.

${ }^{14}$ Anders als vielerorts in der alten Kirche, wo mit einer Ekstase des inspirierten Geistes gerechnet wird, etwa bei Enthusiasten wie Montanus und Tertullianus, aber auch katholischerseits wie bei Ambrosius (vgl. Kelly 1977:62).

${ }^{15}$ Prologus in Pentateucho, 35-39.
} 


\section{Die Problematik des Begriffes hebraica veritas}

der Septuaginta, konnten nicht die volle Wahrheit kennen, weil sie vor dem Leiden und der Auferstehung Christi lebten. Daher konnten sie nicht die volle Wahrheit in ihrer Übersetzung wiedergeben. Hieronymus dagegen lebt - wie die Autoren des neuen Testaments ${ }^{16}$ - nach Christi Leiden und Auferstehung und sieht sich selbst also in der Lage, besser als seine Vorgänger übersetzen zu können. Sein Argument ist also grundsätzlich ausgerichtet. Sowohl er als auch die Siebzig hatten den hebräischen Text zu übersetzen; sie aber von einer Position der Unkenntnis und des "Hörensagens" aus, aufgrund deren nur eine zweifelhafte Übersetzung möglich sei, und er von einer Position der Kenntnis und des sichtbar Wahrgenommenen aus, aufgrund deren eine bessere Übersetzung möglich sei. Daher scheint es inm auch notwendig, die Konsequenz zu ziehen, dass die "Apostel", also die Autoren des Neuen Testaments, den hebräischen Text des Alten Testaments verwendet haben. Das ist das zweite Element der theologischen Grundlage der hebraica veritas: es basiert auf den unterschiedlichen Perspektiven der Epochen vor und nach Christus. Obwohl der Terminus hebraica veritas hier nicht verwendet wird, ist der Begriff da. Die Voraussetzung ist der oben erwähnte pneumatologische Aspekt, nämlich, dass das hebräische Alte Testament inspiriert ist. Ihre Wahrheit ist unangefochten und wird weder von Hieronymus, noch von den Verfechtern der Septuaginta als Alten Testaments der Kirche (etwa Augustin und Rufin) infrage gestellt.

Interessant ist dennoch, dass es Hieronymus in seiner

Argumentation über die Epochen vor und nach Christus nicht nötig erscheint, die Wahrheitsqualität des hebräischen Textes, der ja auch ante adventum Christi, in der Zeit des bloßen "Hörens", geschrieben wurde, vis-à-vis des ante-adventum-Arguments zu verteidigen oder zumindest zu bekräftigen. Eben das fand Augustin notwendig, als er gerade in Auseinandersetzung mit der Vulgata von Hieronymus die Inspiration der Siebzig mit der Inspiration der Hebräisch sprechenden Propheten gleichsetzte (De Civitate Dei XVIII, 43): ${ }^{17}$

\footnotetext{
${ }^{16} \mathrm{Vgl}$. die erste Person Plural (intellegimus, proferimus), wo Hieronymus sonst in den Prologen normalerweise die erste Person Singular in vergleichbaren Kontexten verwendet.

${ }^{17}$ Vgl. oben, § 2.2. Auch Hieronymus scheint anfänglich die Inspiration der Siebzig zugegeben zu haben, etwa im Prolog zur Revision der Vetus Latina nach der Hexapla 8, 14, wo er auf die Siebzig verweist, "qui Spiritu Sancto pleni ea quae vera fuerant transtulerant" ("die vom Heiligen Geiste erfüllt, die Dinge, die wahr sind, übersetzten"; vgl Markschies 1994:144). Dazu konnte er aber unter dem Zugzwang seiner eigenen Argumentführung nicht stehen bleiben.
} 
Spiritus enim, qui in prophetis erat, quando illa dixerunt, idem ipse erat etiam in septuaginta viris, quando illa interpretati sunt; qui profecto auctoritate divina et aliud dicere potuit, tamquam propheta ille utrumque dixisset, quia utrumque idem spiritus diceret, et hoc ipsum aliter, ut, si non eadem verba, idem tamen sensus bene intellegentibus dilucesceret, et aliquid praetermittere et aliquid addere, ut etiam hinc ostenderetur non humanam fuisse in illo opere servitutem, quam verbis debebat interpres, sed divinam potius potestatem, quae mentem replebat et regebat interpretis..

Denn derselbe Geist, der in den Propheten war, als sie diese Dinge sagten, war auch in den siebzig Männern, als sie das übersetzten; und er konnte doch offenbar als Gott mit wirklicher Autorität etwas anderes sagen, als der Prophet ursprünglich gesagt hat, weil derselbe Geist beides sagte; und ebenso konnte, wenn nicht wörtlich, so doch der gleiche Sinn den Verstehenden aufleuchten; er konnte auch etwas weglassen und etwas hinzufügen, so dass auch dadurch gezeigt wird, dass bei dieser Arbeit nicht menschliche Sklaverei als Interpretin einzelner Worte anwesend war, sondern die größere Macht Gottes, die den Geist des einzelnen Übersetzers erfüllte und beherrschte.

Ist also die Inspiration des Geistes im hebräischen Alten Testament und in der Septuaginta gleich, dann ist Hieronymus' heikler Versuch auch überflüssig, das Vorher-Nachher-Schema auf die alten Propheten (zwar vor den Siebzig, aber so inspiriert, dass sie eigentlich auf Christus zurückblicken) und die Siebzig (mangels Inspiration noch immer in ihrer aktuellen Epoche) anzuwenden.

\section{DIE ABGRENZUNGSFUNKTION DER HEBRAICA VERITAS}

Abgesehen von den drei von Hieronymus entwickelten Argumenten zur Begründung der "hebräischen Wahrheit", gibt es innerhalb seiner als Verantwortung gedachten Überlegungen auch mindestens zwei Aspekte, die als Funktionen der hebraica veritas betrachtet werden können. Beide spielen definierende Rollen - einmal sozial, also als Abgrenzung der Kirche gegenüber dem Judentum, und dann literarisch, und zwar als Abgrenzung der kanonischen von den nichtkanonischen Büchern. 


\subsection{Die anti-jüdische Funktion der hebraica veritas}

Logisch stellt sich nun die nächste Frage, nämlich warum die hebraica veritas eine Basis mit den eben erörterten Aspekten braucht. Der Bedarf einer Heiligen Schrift, um das Heilsgeschehen in Christus als Erfüllung der Verheißung an Israel begründen zu können, ist durchaus klar. Aber diese Funktion konnte die Septuaginta übernehmen (und hat sie auch tatsächlich übernommen). Ihre Inspiration stand in der frühen Kirche fest und wurde besonders durch Hieronymus' Gesprächspartner Augustinus behauptet. Warum war denn gerade der hebräische Text nötig? Das geht aus verschiedenen Texten des Hieronymus hervor, ${ }^{18}$ von denen ich stellvertretend zwei zitiere. Im 2. Prolog zu den Psalmen (Zeilen 33-35) heißt es:

Sed quod aliud sit in ecclesiis Christo credentium Psalmos legere, aliud ludaeis singula verba calumniantibus respondere.

Es ist aber eine Sache, die Psalmen in den Kirchen der Christus-Gläubigen zu lesen, eine andere, den Juden zu antworten, die fälschlich jedes Wort verdächtigen.

Im Prolog zum Buch Josua (Zeilen 18-21) wird das Ziel, das Hieronymus vorschwebte, in einer rhetorischen Frage, aber expressis verbis formuliert:

[18] Quae enim audientis [19] utilitas est nos labore sudare et alios detrahendo laborare, dolere ludaeos quod [20] calumniandi eis et inridendi Christianos sit ablata occasio, et Ecclesiae homines id [21] despicere, immo lacerare, unde adversarii torqueantur?

[18] Denn was nutzt es [19] den Hörern, dass wir schwitzend arbeiten und uns anstrengen, andere zu kritisieren, dass den Juden die Gelegenheit verloren gingen, die Christen fälschlich zu verleumden und zu beleidigen, und Männer der Kirche dann das verachten, [21] sogar zerreißen, womit die Gegner gefoltert werden?

Der Sinn der schwierigen Übersetzungsarbeit und der damit verbundenen Kritik ist, dass den Juden die Basis ihrer

\footnotetext{
${ }^{18}$ Verweise bei Sparks (1970:515); Kelly (1975:160); Müller (1989:115-116). Vgl. auch den Prolog zum Buch Daniel (Zeilen 20-25), wo die Verlegenheit von Hieronymus über die Einschließung von Susanna, den Drei Jünglingen sowie Bel und dem Drachen als Teile des Danielbuches klar wird.
} 
ungerechtfertigten Verleumdungen und Beleidigungen genommen wird. Das kann man nur dann machen, wenn man den hebräischen Text zur eigenen Basis macht. Denn man muss einen Text haben, um die Juden widerlegen zu können ${ }^{19}$, und die im Judentum mittlerweile nicht mehr den gleichen Status genießende Septuaginta ist dafür unbrauchbar. Die eigentliche Wurzel der hebraica veritas liegt also in der Auseinandersetzung zwischen Kirche und Synagoge. Diesen Erfolg der hebraica veritas musste sogar Augustinus, der einflussreichste Gegner des Hieronymus, zugeben (De Civitate Dei XVIII, 43). Die Hochschätzung des hebräischen Textes ist keine Hochschätzung des Judentums oder Zuneigung zum Judentum, sondern ein Akt der Aneignung, ein polemisches Unternehmen gegenüber dem Judentum. ${ }^{20}$

Das lässt sich nicht dadurch abmildern, dass Hieronymus sich in Bethlehem niederließ, Juden konsultierte und von innen Hebräisch lernte. Er hat für seinen Hebräischunterricht bezahlt (Ep. 83, 3, 1-2):

Rursum Hierololymae et Bethlehem, quo labore, quo pretio Baraninam nocturnum habui praeceptorem! Timebat enim ludaeos et mihi alterum exhibebat Nicodemus, horum omnium frequenter in opusculis meis facio mentionem.

Wiederum in Jerusalem und Bethlehem - mit welcher Mühe und zu welchem Preis konnte ich Baranina in der Nacht als Lehrer bekommen! Er hatte Angst vor den Juden und zeigte sich mir als einen zweiten Nikodemus. Von alle dem berichte ich des Öfteren in meinen Werken.

Nicht nur hat er sich seine Hebräischkenntnisse einfach erkauft, er berichtet sogar davon in einem unverkennbar judenkritischen Kontext. Vorausgesetzt ist die Spannung zwischen Juden und Christen in Palästina, Baranina (בר־חנינא) wird als ängstlich vorgestellt, und mit etwas Humor im Vergleich ein wenig lächerlich gemacht. ${ }^{21}$

\footnotetext{
${ }^{19}$ Vgl. Sparks (1970:532); Müller (1989:113).

${ }^{20}$ Daher ist auch die euphemistische Formulierung von Markschies, es sei von der "Bibelübersetzung im philologischen Dienst des Gespräches mit Juden" [!] die Rede (1994:154), eine Verharmlosung der antijüdischen Polemik von Hieronymus zu nennen. Zum Glück relativiert Markschies (1994:162) das etwas später, indem er vom "antijüdischen polemischen Vorwurf" im Pentateuch-Prolog spricht.

${ }^{21}$ Obwohl er diesen Text zitiert, zieht Rebenich (1993:61-63) nicht diese Konsequenz. Dafür weist er aber darauf hin, dass Hieronymus' Gegner, Rufin (Apol. c. Hier. 2,15), die Situation voll ausschlachtet (etwa: Baranina = Barabbas!).
} 
Also scheint mir Markschies' Idee, die hebraica veritas spiele "nur eine philologische Rolle" so stark übertrieben, dass sogar ein Kontext, wo Hieronymus' Textuntersuchung unverkennbar anti-jüdische Polemik erhält - in diesem Fall eine Überprüfung von Aquila daraufhin, ob der “ „Haß der Synagoge auf Christus' den Text verändert habe” -, ihm nicht eine Abmilderung nahe legt. ${ }^{22}$

\subsection{Die kanonische Funktion der hebraica veritas}

Eine Seite der Abgrenzung gegenüber dem Judentum besteht darin, dass die neutestamentliche Wahrheit notwendig mit der "hebräischen Wahrheit" begründet werden musste. Den hebräischen Text musste man auch verwenden können, um die neutestamentlichen Zeugnisse als erfüllte Prophetie kennzeichnen zu können. Weil nur der Tanak dafür taugte, wird das hebraica-veritas-Prinzip eine Sache des Umfangs der Offenbarung, also des Kanons (vgl. Müller 110f.). Was sich auf die Inhalte der Texte bezieht, muss notwendig auch Konsequenzen für den Umfang haben. Welche Texte als kanonisch anerkannt werden können und welche nicht, hängt eng mit der inhaltlichen Qualität zusammen. Die nicht hebräisch erlangten Bücher sind separat zu halten und als "Apokryphen" zu betrachten; ${ }^{23}$ sie durften nach Hieronymus zwar gelesen werden, waren aber in der Katechese als ungeschickte Literatur zu vermeiden.

Die Sicht des Hieronymus, die hebraica veritas sei die Bibel des Neuen Testaments gewesen, lässt sich zur Unterstützung dieser These aber nicht aufrechterhalten. Zwar gibt es hebräische und aramäische Wörter im Neuen Testament, aber auf der Ebene des Textes zitiert es mit einigen Ausnahmen aus der Septuaginta, auch wo diese semantisch völlig von der hebräischen Vorlage abweicht - was für die christliche Lehre von fundamentalem Belang sein konnte -, etwa der berühmte Vers aus Jesaja 7,14 (hebräisch: "junge Frau"; Septuaginta: "Jungfrau") als Schriftbeweis für die auf Matthäus 1,23 ("Jungfrau") basierende Lehre der Jungfraugeburt. Diese gut bekannten Fakten passen nun zu einem weiteren wichtigen Gegebenen: Auf der Ebene des Kanons ist die Anordnung des Neuen Testaments nicht dem

\footnotetext{
${ }^{22}$ Markschies (1994:141-142). Das ist umso deutlicher, weil Hieronymus suggeriert, Andeutungen von jüdischem Hass auf Christus gefunden zu haben. Markschies weist selbst auf dieses Faktum hin, sieht darin aber keine Konsequenzen für seine These.

${ }^{23}$ Prolog zum Buch Könige (Zeilen 52-54: "Hic prologus Scripturarum quasi galeatum principium omnibus libris, quos de hebraeo vertimus in latinum, convenire potest, ut scire valeamus, quicquid extra hos est, inter apocrifa seponendum" ("Dieser Prolog zu den Schriften ist als bewaffneter Anfang für alle Bücher, die wir aus dem Hebräischen ins Lateinische übertragen, brauchbar, damit wir wissen können dass das, was außerhalb dieser ist, unter die Apokryphen einzuordnen ist".)
} 
dreiteiligen Aufbau des Tanak, sondern dem vierteiligen der Septuaginta nachgebildet:

I

die 4 grundlegenden Evangelien // die 5 grundlegenden Bücher Mose

II

die Apostelgeschichte //

die geschichtlichen Bücher

III

die Briefe zur Regelung des Gemeindelebens in der Gegenwart //

die Lehrbücher zur Regelung des frommen Lebens in der Gegenwart

IV

die apokalyptische Offenbarung der Zukunft //

die prophetische und apokalyptische Sammlung über die

Zukunft

Fig. 1

Demgegenüber entspricht der Tanak mit seiner Dreiteilung einem völlig anderen Anordnungsprinzip. Hieronymus kennt diese Dreiteilung ${ }^{24}$, konnte seine Auffassung der hebraica veritas in dieser Hinsicht aber nicht durchsetzen. Soweit ich sehe, sind damit zwei Aspekte verbunden: einmal die Reihenfolge und zweitens der Umfang. Der Umfang der Vulgata ist nach den afrikanischen Konzilien von 393, 397

\footnotetext{
${ }^{24}$ Er verweist ausdrücklich darauf im Prolog zum Buch Daniel (Zeilen 45-48), und zwar im Vergleich zur christlichen Aufteilung.
} 
und 419, endgültig aber bereits 405 durch den diesbezüglichen Brief von Papst Innozenz I. ${ }^{25}$ festgelegt worden:
I
II
III

\begin{tabular}{|l|l|l|}
\hline Fünf Bücher Mose & Josua & 5 Bücher des Salomo \\
\hline & Richter & Psalmen \\
\hline & 4 Bücher der Könige & Hiob \\
\hline & Ruth & Tobit \\
\hline & $\begin{array}{l}\text { 16 Bücher der } \\
\text { Propheten }\end{array}$ & Esther \\
\hline & & Judith \\
\hline & & $\begin{array}{l}\text { 2 Bücher der } \\
\text { Makkabäer }\end{array}$ \\
\hline & & 2 Bücher von Esra \\
\hline & & 2 Bücher der Chronik \\
\hline
\end{tabular}

Fig. 2

In dieser Liste (Spalten von mir) spiegelt sich noch etwas von der dreiteiligen hebräischen Kanonstruktur mit Tora, Nebi'im und Ketubim ${ }^{26}$ ab, aber der Umfang ist deutlich nicht mehr der des Tanak. Wie es dazu kam, dass der Umfang schon, die Struktur aber noch nicht fest stand, könnte damit erklärt werden, dass komplette Bibelkodexe noch nicht zu Hieronymus' Zeiten existierten. Der Kanon war eine Liste von normativen Büchern, keine Ausgabe von einem einzigen Kodex. Daher konnte Hieronymus über viele Jahre kreuz und quer durch das, was später der Vulgata-Kanon werden sollte, seine Übersetzungsarbeit treiben, revidieren und überarbeiten. Wie es dazu kam, dass die Reihenfolge, sprich: Aufbau, der vorliegenden Vulgata strukturiert ist wie sie heute aussieht, wissen wir nicht. Sparks ${ }^{27}$ vermutet, dass die Arbeit von Cassiodor von Scylacium (Ende 6. Jh.) dazu führte, weil wir von der Vulgata als "Pandektes" zum ersten Mal in Zusammenhang mit

\footnotetext{
${ }^{25}$ Innozenz I., Ep. 6,7.

${ }^{26}$ Diese Einteilung hat Hieronymus gekannt, wie aus seinem Prolog zu den Königsbüchern (Zeilen 21-45) ersichtlich ist.

${ }^{27}$ Sparks 1970:521-522.
} 
seiner Bibliothek hören. Ein Pandektes ${ }^{28}$ kann aber entweder eine umfangreiche Sammlung oder eine komplette Gesetzeskodifizierung bedeuten. Nun heißt es in Cassiodors Institutiones divinarum et humanarum litterarum I, 12,3:

Hunc autem pandectem propter copiam lectionis minutiore manu in senionibus quinquaginta tribus aestimavimus conscribendum, ut quod lectio copiosa tetendit scripturae densitas adunata contraheret.

Nun, denken wir, ist diese Sammlung wegen der Vielfalt des Lesens mit kleinerer Hand in 50 sechsfächig geteilten Behältern zusammenzuschreiben, damit - weil vielfältige Lesung dazu tendierte - die Dichte der Schrift die unfassbaren Dinge zusammen bündelt.

Vergleichen wir nun das unten stehende Bild von Cassiodor ${ }^{29}$ als Esra der Sopher, dann kann eine gute Vorstellung von der Bibel als Pandektes gemacht werden. Die Bibel als Sammlung war zwar zusammengebracht, aber noch immer kein einziges Buch.

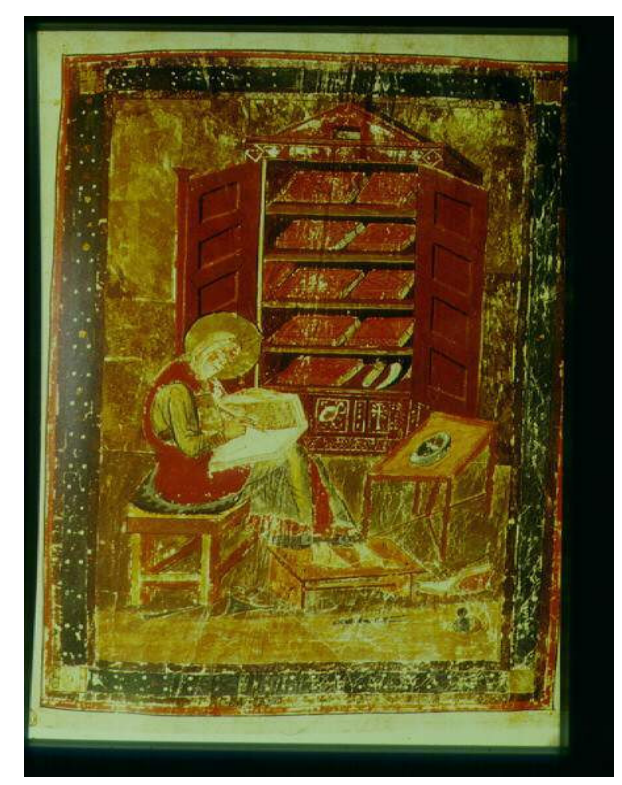

Fig. 3: Codex Amiatinus, 8. Jh.: Cassiodor als Esra

\footnotetext{
${ }^{28}$ Latein: pandectes, "Enzyklopädie", aus Griechisch $\pi \alpha \nu \delta \epsilon \kappa \tau \eta \zeta$, alleserhaltend: $\pi \alpha \nu-$,

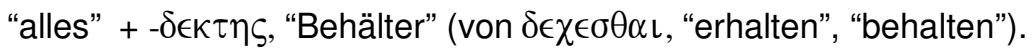

${ }^{29}$ Die Bücher im Schrank, von denen einige Titel lesbar sind, suggerieren Bibelbücher, die laut Cassiodors Institutiones in seiner Bibliothek anwesend war.
} 


\section{Die Problematik des Begriffes hebraica veritas}

Demgemäß lassen sich sowohl die Variationen in der Reihenfolge als auch die relative Stabilität des Umfangs erklären. Für Hieronymus war die hebraica veritas eine Liste von mehreren kanonischen Büchern. Aber er hatte sich nicht gescheut, auch nichthebräische Bücher ins Lateinische zu übersetzen, und er hat die Vetus Latina anhand der hexaplarischen Septuaginta revidiert, so dass es nicht wundert, dass sein hebraica-veritas-Prinzip sich nicht im Kanon der spätantiken und mittelalterlichen Kirche durchsetzte. Ab dem 8. Jh. besteht die Vulgata aus seinen Übersetzungen aus dem Hebräischen (ohne Psalmen), seinem "Gallikanischen Psalter", seinen Übersetzungen von Tobit und Judith, Büchern aus der Vetus Latina (Sapientia, Ecclesiasticus, 1. und 2. Makkabäer, Baruch), seinen Revisionen der Evangelien, und aus Revisionen der Apostelgeschichte, der Briefe und der Offenbarung durch Unbekannte. Diese "Vulgata" ist also keine lautere Widerspiegelung des hebraica-veritas-Prinzips, das so energisch von Hieronymus verfochten wurde. Der Aufbau der Vulgata wurde ein Abbild der vierteiligen Gruppierung in der griechischen Tradition, die auch im Neuen Testament nachgebildet wurde und allmählich von einer Gruppierung von mehreren Büchern zur Struktur eines einzigen großen gebundenen Buches überging. Dazu war die Erfindung des Kodex der endgültige Impuls. De facto ist das Prinzip der hebraica veritas damit auch untergegangen und die Vulgata in dieser Form wurde die "Katholische Bibel". Als solche wurde sie aber auch für die Reformation und die nachreformatorische Orthodoxie zum willkommen Angriffspunkt.

\section{IMPLIKATIONEN DER PROBLEMATIK SEIT DER REFORMATION}

Das Prinzip der "hebräischen Wahrheit" war ein theologischer Versuch, christlich mit dem Alten Testament umzugehen. Das bedeutete, dass Christus der Mittelpunkt wurde, wie aus der Vorher-NachherHermeneutik ${ }^{30}$ hervorgeht, wofür die Inspiration ${ }^{31}$ das vertraute Mittel war. Daher rückt die Beschäftigung mit der hebraica veritas von Hieronymus natürlich Fragen nach deren Implikationen für die christliche Theologie in den Vordergrund, angesichts der Zentralität des Kanonbegriffes bei Hieronymus, in der rezenten Bibelwissenschaft und

\footnotetext{
${ }^{30}$ Der heilshistorischen Aspekt, oben unter $\S 2.3$ besprochen.

${ }^{31} \mathrm{Vgl}$. oben, § 2.3 .
} 
besonders während der jüngsten zwei Dekaden nicht zuletzt die Kanonfrage. Als Schlussüberlegung seien einige dieser Aspekte hier programmatisch angeschnitten.

\subsection{Die polemische Verwendung der hebraica veritas durch die Reformation.}

Der Protestantismus, ${ }^{32}$ konnte sich der hebraica veritas als antikatholischer Polemik bedienen. Die hebräische Wahrheit war damals und bleibt bis heute eine oft unausgesprochene wie undurchdachte Voraussetzung der christlichen Exegese des Alten Testaments. Der protestantische Kanon besteht im Alten Testament nur aus den Büchern, die im hebräischen Tanak vorkommen. Nur was hebräisch vorlag, konnte kanonisch sein, allen anderen Büchern der "Katholischen Bibel" wird der kanonische Status aufgrund der hebraica veritas abgesprochen. Aber die Reformatoren haben einen hybriden Kanon geschaffen, also einen Kanon, den es vorher noch nie gegeben hat und seitdem auch nur in nationalen Übersetzungen gibt. In der Luther-Übersetzung und in parallelen Übersetzungen in Volkssprachen wie die berühmten englischen und niederländischen "vulgaten" Bibeln wurde, vom Umfang abgesehen, genau das gemacht, was in der katholischen Vulgata erreicht wurde: ein neuer Kanon als Berufungsgrund für die Verteidigung gegenüber einer anderen Religionsgemeinschaft. Die Zueignung des hebräischen Kanons durch Hieronymus war eine antijüdische Polemik, die Zueignung desselben in der reformatorischen Tradition eine antikatholische.

\subsection{Die Struktur des hebräischen Tanak hatte hermeneutisch wenig Effekt}

Die Dreiteilung der hebräischen Bibel in Tora, Nebi'im und Ketubim gehört als sein Bauprinzip zum Wesen des Tanak. Weil Form literarisch alles mit Inhalt zu tun hat, hat diese Struktur auch alles mit Inhalt und Sinn der hebräischen Bibel zu tun. Logischerweise wäre zu erwarten, dass sie auch zum Wesen jedweder Verwendung des Konzepts

\footnotetext{
${ }^{32}$ Frühere Fachliteratur und Belege (Luther, Oekolampad) bei Hempelmann (1989:47). Hempelmanns eigener Beitrag basiert aber auf einen sprachphilosophischen "Abschied von einer cartesianischen Linguistik" zugunsten einer Sprachphilosophie, "daß eine jede Sprache eine spezifische 'Einteilung von Welt' und damit eine (Mit-) Konstitution von Wirklichkeit bedeutet, die nicht von dieser als 'Ontologie' oder 'Metaphysik' ablösbar ist” (1989:40-41). Diesem Thema wird hier nicht weiter nachgegangen, abgesehen nur von einem Hinweis auf meine Skepsis gegenüber dem philosophischen Ansatz, nach dem (in diesem Fall) die hebraica veritas die in der hebräischen Sprache inhärenten Qualitäten bedeuten sollte, etwa semantische und syntaktische Möglichkeiten, die Wirklichkeit "wahrhaft" zum Ausdruck zu bringen (vgl. Hempelmann 1989:48).
} 


\section{Die Problematik des Begriffes hebraica veritas}

hebraica veritas gehören würde. Das war jedoch nie der Fall. Obwohl die Idee der hebraica veritas sich ausdrücklich auf die rabbinischjüdische Überlieferung berief, und obwohl Hieronymus den "Apokryphen" im Geiste des Tanak reserviert gegenüber stand und die inm augenscheinlich folgende protestantische Tradition die "Apokryphen" tatsächlich ausklammerte, hat die rabbinisch-jüdische Sicht der hebräischen Wahrheit sie nicht beeinflusst. Wenn überhaupt, scheint die Zueignung der hebräischen Bibel als Heiliger Schrift des Christentums sich nur in Ausnahmefällen für die hermeneutischen Implikationen sämtlicher Aspekte der hebraica veritas, einschließlich ihrer übergreifenden Textorganisation und Struktur, zu interessieren. Das ist in der protestantischen Tradition von Hochachtung für die hebraica veritas nicht zu erwarten.

\subsection{Einen Kanon von hebräischem AT und griechischem NT gibt es nicht}

Einen Kanon, der aus dem hebräischen Alten Testament und dem griechischen Neuen Testament besteht, gibt es nicht und hat es nie gegeben. Die aus hebräischem Umfang und griechischer Struktur gemischte, aber in einer dritten Sprache wiedergegebene Form des protestantischen Kanons ist teilweise eine Analogie von dem, was Hieronymus zustande gebracht hatte. Zunächst wurde sein Ideal der hebraica veritas bereits in der Vulgata sowohl in Aufbau als auch in Umfang mit der von ihm selbst abgelehnten Septuaginta-Überlieferung verbunden. Er konnte das Ideal nicht durchsetzen, während die Reformatoren das nur hinsichtlich des kanonischen Umfangs konnten. Ironischerweise liegt ein hebräischer Kanon in keinem der beiden Fälle vor - weder in der Vulgata noch im protestantischen Kanon. In der protestantischen Theologie wird Jahrhunderte lang einerseits unkritisch vorausgesetzt, man ginge so von einem gegebenen Kanon als einer Bibel, also einem Buch aus. Andererseits kann man bei der Inkonsequenz von einer Verbindung zwischen hebraica veritas und griechischem Neuen Testament bestenfalls vom Kanon als Liste oder Pandektes sprechen, keinesfalls aber von einem Kanon als Buch.

\subsection{Intertextuelle Begrenzung und Ergänzung}

Die hebräisch- und griechisch-jüdischen Traditionen der

Bibelüberlieferung kristallisierten sich in sehr unterschiedliche

Buchformen und manifestierten unterschiedliche religiöse

Überzeugungen. Historisch gilt das schon innerhalb des Judentums, da 
beide Formen jüdisch sind. Gerade so ist die Thematik, für die der Begriff hebraica veritas Chiffre wurde, für die theologische Verwendung des Alten Testaments von erheblichem Gewicht. Auch jenem, der Hieronymus nicht zustimmen kann, ist damit die Frage nach dem legitimen Text des Alten Testaments gestellt. Ihre Beantwortung ist sowohl für die Bestimmung des auszulegenden Textes als auch für die Gestalt des christlichen Alten Testaments ausschlaggebend. Dabei ist irrelevant, ob Sorge um die Übersetzungsqualität und vielförmige Überlieferung der Septuaginta oder um den in ihr vorhandenen Überschuss an damals nicht hebräisch vorliegenden "Apokryphen" Anlass zur Bevorzugung der hebräischen Bibel ist - Umfang sowie Struktur des Kanons sind zum theologischen Problem geworden. Dieses Problem scheint mir eher dadurch bewältigt werden zu können, dass das literarische Phänomen der Intertextualität ernst genommen wird. Das beinhaltet unter vielen anderen auch den Respekt für die gegenseitige Begrenzung und daher bereichernde Ergänzung, die verschiedene Textüberlieferungen und -organisationen mit sich bringen. Eine Illustration bietet etwa die Möglichkeiten des gleichermaßen von dem hebraica-veritas- und dem Vulgata-Aufbau geprägten protestantischen Schriftprinzips: es lebt aus zwei Traditionen, nach denen etwa Prophetie jeweils etwas Verschiedenes bedeutet. Einerseits ist die Prophetie in der hebräischen Tradition Gottes Begleitung der Gegenwart seines Volkes im Lichte der Geschichte. Andererseits ist sie aber in der Septuaginta-VulgataTradition Vorhersage der Zukunft. Daher sind in der hebräischen Textorganisation die "geschichtlichen" und "prophetischen" Bücher gemeinsam als die "Vorderen" und "Hinteren" Propheten zusammengebracht. In der Septuaginta-Vulgata-Tradition gehören andererseits die prophetischen und apokalyptischen Bücher als Weissagungen der Zukunft nach den historischen Büchern zusammen. Der protestantische Kanon des Alten Testaments ist nur dann keine Hybride, wenn er Zugang zum Reichtum seiner beiden Herkunftsstränge wird. Diese Implikation sprengt aber den Rahmen des hier gebotenen Aufsatzes und verdient eine eigene Behandlung.

\subsection{Inspirationsauffassungen}

Umgang mit dem Begriff hebraica veritas hat auch für die systematische Theologie Bedeutung. Das Prinzip wurde in der nachreformatorisch-protestantischen Theologie die alttestamentliche Norm zur Bestimmung, Begründung und Bestätigung der christlichen 
Lehre. Ihre Verwendung führt aber verschiedene Probleme herbei. Zum einen setzt sie einen statischen Text, den es einmal gegeben hat, voraus, der wieder gegenüber allen anderen Textformen abzusichern und für Lehre, Theologie und Verkündigung zu verwenden sei. Da dieser Text als solcher inspiriert sei, ist die dahinter liegende Inspirationsauffassung auch statisch und einer mechanistischen Schriftanschauung ausgesetzt. Anders umformuliert: wenn eine statische Inspirationstheorie zu vermeiden ist, ist eine statische Kanonauffassung ebenso zu vermeiden, und dafür ist ein neues Durchdenken des systematisch-theologischen Umgangs mit dem Kanon nötig.

\section{6 "Gesamtbiblische" Theologien}

Darüber hinaus stellt sich die Frage, ob das neuerdings entwickelte Konzept einer "gesamtbiblischen Theologie" des Alten und Neuen Testaments überhaupt realisierbar ist, wenn man sich der Idee der hebraica veritas im Sinne des Hieronymus gegenüber der Septuaginta/Vulgata anschlie $3 t{ }^{33}$ Demgegenüber ist auch versucht worden, nicht sosehr den Fragen der Textgestalt und Kanonizität als vielmehr der "hebräischen Sprachgestalt der biblischen Offenbarung" systematisch-theologisch gerecht zu werden - was aber mit der hebraica veritas im eigentlichen Sinne nichts mehr zu tun hat.

Obwohl es den Rahmen dieses Aufsatzes sprengen würde, sollten diese Fragen - zumal damit bereits ein Beginn gemacht wurde ${ }^{34}$ - im Bereich der "alttestamentlichen", der "biblischen" und der "systematischen" Theologie im Lichte der Tradition neu aufgegriffen und weiter aufgearbeitet werden.

\section{Bibliographie}

Bammel, C P 1988. Die Hexapla des Origenes: Die "hebraica veritas" im Streit der Meinungen. Augustinianum 28, 125-147.

Bartelink, G J M 1980 . Hieronymus: Liber de optimo genere interpretandi (Epistula 57). Ein Kommentar. Leiden: Brill. (Mnemosyne Suppl 61.)

\footnotetext{
${ }^{33}$ Vgl. z.B. das Paradebeispiel dieser Tendenz von Brevard S. Childs unter dem deutschen Titel Die Theologie der einen Bibel, dessen englischen Originaltitel kennzeichnend ist: Biblical Theology of the Old and New Testament: Theological Reflexion on the Christian Bible.

${ }^{34}$ Vgl. die Arbeiten von Haacker \& Hempelmann (1989), Hobbs (1984); auch zu vergleichen wäre Willi (2002) (mit sprachlichem Fokus).
} 
Childs, B S 1994-1996. Die Theologie der einen Bibel. Bd 1 \& 2. Freiburg: Herder. [Aus dem Englischen: Biblical Theology of the Old and New Testament: Theological Reflexion on the Christian Bible . London: SCM 1992].

Haacker, K \& Hempelmann, H 1989. Hebraica Veritas: Die hebräische Grundlage der biblischen Theologie als exegetische und systematische Aufgabe. Wuppertal: R Brockhaus.

Hempelmann, H 1989. Veritas hebraica als Grundlage christlicher Theologie. Zur systematisch-theologischen Relevanz der biblisch-hebräischen Sprachgestalt, in Haacker \& Hempelmann 1989:39-78.

Hengel, M\& Schwemer, A M (Hg) 1994. Die Septuaginta zwischen Judentum und Christentum. Tübingen: J C B Mohr. (WUNT 72.)

Hobbs, R G 1984. Hebraica Veritas and Traditio Apostolica: Saint Paul and the Interpretation of the Psalms in the Sixteenth Century, in Steinmetz (ed) 1984:83-99.

Kelly, J N D 1975 Jerome: His life, writings, and controversies. New York: Harper \& Row.

Lemaire, A (ed) 2002. Congress Volume: Basle 2001. Leiden: Brill. (Vetus Testamentum Supplements 92.)

Markschies, C 1994. Hieronymus und die "Hebraica Veritas" - ein Beitrag zur Archäologie des protestantischen Schriftverständnisses, in Hengel \& Schwemer 1994:131-181.

Müller, M 1989. Graeca sive hebraica veritas? The defence of the Septuagint in the early church. SJOT 1989, 103-124.

Rebenich, S 1993. Jerome: The "Vir Trilinguis" and the "Hebraica Veritas". Vigillae Christianae 47, 50-77.

Sparks, H F D 1970. Jerome as biblical scholar, in The Cambridge History of the Bible 1, 510-541. Cambridge: CUP.

Steinmetz, D C (ed) 1984. The Bible in the sixteenth century. Durham, NC: Duke University Press. (Duke Monographs in Medieval and Renaissance Studies 11.)

Strack, H \& Billerbeck, P 1926-1928. Kommentar zum Neuen Testament aus Talmud und Midrasch. München: Beck.

Willi, T 2002. Hebraica Veritas in Basel: Christliche Hebraistik aus jüdischen Quellen, in Lemaire (ed) 2002:375-398. 